\title{
Serum Anti-Gal-3 Autoantibody is a Predictive Marker of the Efficacy of Platinum-Based Chemotherapy against Pulmonary Adenocarcinoma
}

\author{
Kengo Yanagita ${ }^{1}$, Ryo Nagashio ${ }^{1}$, Shinichiro Ryuge ${ }^{2}$, Ken Katono ${ }^{2}$, Shi-Xu Jiang ${ }^{3}$, \\ Benio Tsuchiya ${ }^{1}$, Hiroyasu Nakashima ${ }^{4}$, Eriko Fukuda ${ }^{5}$, Naoki Goshima $^{5}$, Makoto \\ Saegusa $^{3}$, Yukitoshi Satoh ${ }^{4}$, Noriyuki Masuda ${ }^{2}$, Yuichi Sato ${ }^{1 *}$
}

\begin{abstract}
Background: Identification of predictive markers for the efficacy of platinum-based chemotherapy is necessary to improve the quality of the life of cancer patients. Materials and Methods: We detected proteins recognized by autoantibodies in pretreated sera from patients with lung adenocarcinoma (AC) evaluated as showing progressive disease (PD) or a partial response (PR) after cisplatin-based chemotherapy by proteomic analysis. Then, the levels of the candidate autoantibodies in the pretreated serum were validated by dot-blot analysis for $22 \mathrm{AC}$ patients who received platinum-based chemotherapy, and the expression of identified proteins was immunohistochemically analyzed in $40 \mathrm{AC}$ biopsy specimens. Results: An autoantibody against galectin-3 (Gal-3) was detected in pretreated sera from an AC patient with PD. Serum IgG levels of anti-Gal-3 autoantibody were significantly higher in patients evaluated with PD than in those with PR and stable disease (SD) $(p=0.0084)$. Furthermore, pretreated biopsy specimens taken from patients evaluated as showing PD following platinumbased chemotherapy showed a tendency to have a higher positive rate of Gal-3 than those with PR and SD ( $p=$ 0.0601). Conclusions: These results suggest that serum IgG levels of anti-Gal-3 autoantibody may be useful to predict the efficacy of platinum-based chemotherapy for patients with lung AC.
\end{abstract}

Keywords: Galectin-3 - autoantibody - lung adenocarcinoma - predictive marker - 2Dß gel electrophoresis

Asian Pac J Cancer Prev, 16 (17), 7959-7965

\section{Introduction}

Lung cancer is one of the most common neoplasms worldwide. It is the leading cause of cancer-related death in Japan, and the 5-year overall survival rate is still below $16 \%$. Non-small cell lung cancer (NSCLC) accounts for approximately $80 \%$ of all lung cancers, and adenocarcinoma (AC) comprises about $50 \%$ of NSCLC (Jemal et al., 2007). Although platinum-based chemotherapy regimens improve the survival of patients with advanced NSCLC, resistance to chemotherapeutic agents is a major problem (Watanabe et al., 2003, Perng et al., 2008). In clinical practice, the chemotherapy response is variable among individuals. About one-third of NSCLC patients achieve complete remission (CR) or a partial response (PR) following the standard first-line chemotherapy, while another one-third show stable disease (SD) and progressive disease (PD), respectively (Chang, 2011). Thus, the identification of predictive markers of the response to chemotherapy is an urgent issue.
Autoantibodies are generally produced in sera of patients with various autoimmune diseases. They are also frequently observed in sera of patients with various neoplasms, even in the early stages. Therefore, the possibilities of using them as potential tumor markers have been suggested (Naour et al., 2002; Fernández-Madrid et al., 2004; Xia et al., 2005). Hanash (Hanash, 2003) reported that analyzing the immune response to identify novel cancer biomarkers is an attractive strategy, because the immune system induces biological amplification that is equivalent to a polymerase chain reaction (PCR) by generating a detectable signal with antigenic tumor proteins as templates, beginning at a very early stage during tumor development before the tumor identifiable. Some tumor-associated autoantibodies have also been reported for lung cancer (Vural et al., 2005; Yagihashi et al., 2005).

Cis-diamino-dichloroplatinum (II) (cisplatin) is a common agent used for chemotherapy against various cancers, including lung cancer. Cisplatin is a cytotoxic

${ }^{1}$ Department of Applied Tumor Pathology, Graduate School of Medical Sciences, Departments of ${ }^{2}$ Respiratory Medicine, ${ }^{3}$ Pathology, and ${ }^{4}$ Thoracic and Cardiovascular Surgery, School of Medicine, Kitasato University, Kanagawa, ${ }^{5}$ Molecular Profiling Research Center for Drug Discovery, National Institute of Advanced Industrial Science and Technology, Tokyo, Japan *For correspondence: yuichi@med.kitasato-u.ac.jp 
compound, which inhibits transcription and DNA replication, and induces apoptosis (Gonzalez et al., 2001). For lung cancer patients, cisplatin was found to be more effective than radiotherapy, and the combination of cisplatin and vinorelbine improved survival (Pepe et al., 2007). Multiple mechanisms have been suggested to explain platinum resistance, such as decreased tumor blood flow, altered extracellular conditions, reduced platinum uptake, increased efflux, intracellular detoxification by glutathione or decreased binding, DNA repair, decreased mismatch repair, defective apoptosis, the activation of anti-apoptotic factors or other signaling pathways, or the presence of quiescent non-cycling cells (Stewart, 2007). The mechanisms underlying cisplatin resistance have not been fully elucidated, and response-predictive markers of cisplatin have not been identified, which are clinical issues.

In this study, we searched for novel predictive markers of the therapeutic effect of cisplatin by two-dimensional immunoblotting (2DE-IB) with pretreated sera from patients who showed a partial response (PR) or progressive disease (PD) after platinum-based chemotherapy, and found that anti-Galectin-3 (Gal-3) autoantibody was correlated with the efficacy of the response to platinumbased chemotherapy.

\section{Materials and Methods}

\section{Cell lines}

The LC2/ad cell line derived from lung AC was purchased from RIKEN BioResource Center (Ibaraki, Japan). LC2/ad cells were grown in RPMI-1640 medium (SIGMA, Steinheim, Germany) supplemented with $10 \%$ heat-inactivated fetal bovine serum (Biowest, Miami, FL, USA), 100 units/mL penicillin, and $100 \mu \mathrm{g} / \mathrm{mL}$ streptomycin (Life Technologies Corp., Carlsbad, CA, USA). Cells were kept at $37^{\circ} \mathrm{C}$ in a humidified atmosphere of $5 \% \mathrm{CO}_{2}$ and $95 \%$ air. Harvested cells were washed twice with phosphate-buffered saline without bivalent ions (PBS-) and stored at $-80^{\circ} \mathrm{C}$.

A cisplatin-resistant sub-line (LC2/ad-cis) was previously established and stably grown with a concentration of $3,200 \mathrm{ng} / \mathrm{mL}$ cisplatin for over 12 months in our laboratory (Kageyama et al., 2011). After harvesting and washing twice with PBS-, LC2/ad-cis cells were stored at $-80^{\circ} \mathrm{C}$.

\section{Serum and tissue samples}

The pre-treated sera from 22 patients with lung AC, who received platinum-based chemotherapy at Kitasato

Table 1. Combinations of Platinum-based Chemotherapy in Patients in this Study

\begin{tabular}{lcccc}
\hline Regimen & $\mathrm{n}$ & \multicolumn{3}{c}{ Response } \\
& & PR & SD & PD \\
\hline CDDP+GEM & 7 & 2 & 3 & 2 \\
CDDP+DOC & 1 & 1 & 0 & 0 \\
CBDCA+GEM & 3 & 0 & 0 & 3 \\
CBDCA+DOC & 2 & 0 & 1 & 1 \\
CBDCA+PTX & 9 & 4 & 2 & 3 \\
\hline
\end{tabular}

CDDP, cisplatin; CBDCA, Carboplatin; GEM, gemcitabine; DOC, docetaxel; PTX, paclitaxel
University Hospital, were collected and stored at $-80^{\circ} \mathrm{C}$ until use. The number of underwent platinum-based chemotherapy and responses are shown in Table 1. Eight patients received cisplatin-based chemotherapy, including gemcitabine for 7 patients and docetaxel for the remaining one. Fourteen patients were treated with carboplatin-based chemotherapy, including 3 with gemcitabine, 2 with docetaxel, and 9 with paclitaxel. The responses to the chemotherapies were assessed by RECIST (version 1. 1), and as shown in Table 1, 7 patients showed PR, 6 showed $\mathrm{SD}$, and 9 showed PD.

Pre-treatment biopsy samples from 40 lung AC patients who received platinum-based chemotherapy, at Kitasato University Hospital were used in this study. Among the 40 patients, 30 were treated with cisplatin-based regimens including 22 with gemcitabine, 7 with irinotecan, and 1 with etoposide. The remaining 10 patients were treated with carboplatin-based chemotherapy: 8 with paclitaxel and 2 with etoposide. The responses to the chemotherapies were assessed as PR in 11, SD in 14, and PD in 15 patients.

All samples were collected in accordance with the ethical guidelines and written consent mandated, and this study was approved by the Ethics Committee of Kitasato University School of Medicine (B07-06). All patients were approached based on approved ethical guidelines, and all agreed to participate in this study and provided written consent. Patients could refuse entry and discontinue participation at any time.

\section{Two-dimensional gel electrophoresis-immunoblotting}

Two-dimensional gel electrophoresis with agarose (agarose 2-DE) method was performed with minor modifications to make it suitable for mini-gel (Nagashio et al., 2008). In brief, LC2/ad-cis cells were solubilized in lysis buffer [7 M urea, $2 \mathrm{M}$ thiourea, 2\% 3-[(3-cholamidopropyl) dimethylammonio] propanesulfonic acid, $0.1 \mathrm{M}$ dithiothreitol, 2.5\% pH 3-10 Pharmalyte (GE Healthcare Bio-Sciences, Piscataway, NJ, USA), and 0.1 tablet $/ \mathrm{mL}$ of complete mini EDTA-free protease inhibitors (Roche Diagnostics, Mannheim, Germany)] using an ultrasonic homogenizer (VP-050; TAITEC, Saitama, Japan), and centrifuged at $20,000 \mathrm{xg}$ for $30 \mathrm{~min}$ at $4^{\circ} \mathrm{C}$. The protein was performed using 2-D Clean-Up Kit (GE Healthcare). The first-dimensional agarose isoelectric focusing (IEF) gel (80 mm long, 2.5-mm inner diameter) was made using a single $\mathrm{pH}$ 3-10 pharmalyte.

The extracted proteins were applied to the cathodic end of the agarose IEF gel, and loaded at $4^{\circ} \mathrm{C}$ in stepwise voltages $(1 \mathrm{hr}$ at $100 \mathrm{~V}, 1 \mathrm{hr}$ at $300 \mathrm{~V}, 1 \mathrm{hr}$ at $500 \mathrm{~V}, 2$ hrs at $700 \mathrm{~V}$, and $3 \mathrm{hrs}$ at $900 \mathrm{~V}$ ). Then, the gels were immersed in trichloroacetic acid (TCA)-fixative solution (10\% trichloroacetic acid, 5\% sulfosalicylic acid) for $3 \mathrm{~min}$ at room temperature (RT) with shaking. After rinsing in distilled water (DW) three times for $15 \mathrm{~min}$ each, gels were treated in sodium dodecylsulfate (SDS) solutions (0.05 M Tris- $\mathrm{HCl} \mathrm{pH} 6.8,2 \%$ sodium dodecyl sulfate, $10 \%$ glycerol, $5 \%$ mercaptoethanol, and $0.02 \%$ bromophenol blue) for $15 \mathrm{~min}$ at RT with mild shaking. Then, each agarose gel was placed on the top of the second dimensional SDS-polyacrylamide gel electrophoresis (SDS-PAGE) gel with $12 \%$ polyacrylamide gel (7-9 
Serum anti-Gal-3 Antibodies for Prediction of Efficacy of Platinum-based Chemotherapy against Pulmonary Adenocarcinoma $\mathrm{cm})$, and loaded with a constant current at $20 \mathrm{~mA}$. Two pieces of gel were prepared: one was for protein transfer to a polyvinylidene difluoride membrane (Millipore, Bedford, MA, USA) for immunoblotting, and the other was stained with coomassie brilliant blue R-350 (CBB) solution (PhastGel Blue R; GE Healthcare).

After blocking with $0.5 \%$ casein $/ 0.01 \mathrm{M}$ Tris- $\mathrm{HCl}, \mathrm{pH}$ $7.5,150 \mathrm{mM} \mathrm{NaCl}$ (TBS) for $60 \mathrm{~min}$ at RT, the membranes were reacted with 100-times-diluted pre-treated sera from three lung AC patients who were assessed as showing PR or PD after platinum-based chemotherapy with $0.5 \%$ casein/TBS-T (TBS containing $0.1 \%$ Tween 20 ) overnight at RT. Then, the membranes were reacted with 1,000-times-diluted horseradish peroxidase (HRP)conjugated rabbit anti-human IgG polyclonal antibody (Dako, Glostrup, Denmark) with $0.5 \%$ casein/TBS-T for $30 \mathrm{~min}$ at RT. Finally, immunoreactive spots on the membranes were visualized with the Stable DAB solution (Lifetechnologies Corp.).

\section{Identification of proteins recognized by autoantibodies}

The protein spots matched with the immunoreactive spots were manually excised from CBB-stained 2-DE gels and destained with $50 \%$ acetonitrile $(\mathrm{ACN}) / 50$ $\mathrm{mM}$ NH4HCO3. The pieces of gel were dehydrated with $100 \%$ ACN and dried under vacuum conditions. Continuously, they were rehydrated in $10 \mu \mathrm{L}$ of digestion solution containing $10 \mathrm{ng} / \mu \mathrm{L}$ trypsin (Trypsin Gold, Mass Spectrometry Grade, Promega, Madison, WI, USA) for $45 \mathrm{~min}$ at $4^{\circ} \mathrm{C}$, and then incubated for $24 \mathrm{hrs}$ at $37^{\circ} \mathrm{C}$ with a minimum volume of $25 \mathrm{mM} \mathrm{NH} 4 \mathrm{HCO} 3$. After incubation, digested tryptic peptide solutions were collected, and the gel was washed once with $50 \%$ $\mathrm{ACN} / 5 \%$ trifluoroacetic acid and collected in the same tube. Solutions containing digested tryptic peptide were then subjected to peptide mass fingerprint (PMF) and MS/ MS analyses for protein identification with autoflex III matrix assisted laser desorption ionization-time of flight/ time of flight mass spectrometry (MALDI-TOF/TOF MS, Bruker Daltonics GmbH, Bremen, Germany). The PMF and MS/MS spectra were submitted to MASCOT (http:// www.matrixscience.com/) for a database search, and the identification of corresponding proteins was conducted with the following database: IPI human database version 3.85 (89,952 sequences; 36,291,020 residues, http://www. ebi.ac.uk/IPI/IPIhuman.).

\section{One-dimensional gel electrophoresis immunoblotting}

Proteins were extracted from lung cancer cell lines with detergent lysis buffer (Laemmli, 1970) using an ultra-sonic homogenizer (VP-050; TAITEC). Each 10 $\mu \mathrm{g}$ of extracted proteins was boiled and separated by SDS-PAGE using $10 \%$ gel with a constant current at 20 $\mathrm{mA}$. After being transferred onto a PVDF membrane and blocked with $0.5 \%$ casein/TBS-T for $60 \mathrm{~min}$ at RT, the membranes were reacted with 500-times-diluted antiGal-3 antibody (Leica Biosystems, Milton Keynes, UK) with $0.5 \%$ casein/TBS-T for $2 \mathrm{hrs}$ at RT. The membranes were continuously reacted with 1,000-times-diluted HRPconjugated rabbit anti-mouse IgG polyclonal antibody (Dako) with $0.5 \%$ casein/TBS for $30 \mathrm{~min}$ at RT. Finally,

immunoreactive bands on the membranes were detected with Chemiluminescent HRP Substrate (Millipore Corp.), and captured with ATTO Cool Saver System (ATTO, Tokyo, Japan).

\section{Immunohistochemistry}

Ten percent formalin-fixed and paraffin-embedded lung cancer cell lines and lung AC biopsy specimens were used for IHC. Three-micrometer-thick sections were deparaffinized in xylene and rehydrated in a descending ethanol series, and then treated with $3 \%$ hydrogen peroxide for $10 \mathrm{~min}$. After blocking with $2 \%$ normal swine serum (NSS)/TBS for $10 \mathrm{~min}$ at RT, the sections were reacted with 200-times-diluted anti-Gal-3 antibody with $2 \% \mathrm{NSS} / \mathrm{TBS}$ for $16-18 \mathrm{hrs}$ at RT. Then, the sections were reacted with ChemMate ENVISION reagent (Dako) for $30 \mathrm{~min}$ at RT. Finally, the sections were visualized using Stable DAB solution (Invitrogen) and counterstained with Mayer's hematoxylin.

\section{Evaluation of immunohistchemistry}

Cytoplasmic immunostaining of tumor cells was considered to be a positive result for anti-Gal-3 autoantibody. The staining intensity was categorized into four groups: $0=$ negative; $1=$ weakly positive; $2=$ moderately positive, $3=$ strongly positive. The tumor cells with staining scores of 2 and 3 were judged as positive. The $\mathrm{X}^{2}$-test was used for the statistical evaluation of staining data. $\mathrm{p}<0.05$ was considered significant.

\section{Preparation of Gal-3 protein for dot-blot analysis}

i) Electro-elution method: Protein extraction from the CBB-stained 2-DE gels were performed using Mini GeBA (Gene Bio-Application Ltd., Yavne, Israel) according to the manufacturer's instructions. In brief, a Gal-3 protein spot was excised from 2-DE gel and transferred to a Mini GeBAflex-tube. After being filled with proteinrunning buffer (0.192 M glycine, $0.025 \mathrm{M}$ Tris- $\mathrm{HCl} \mathrm{pH}$ 7.5 , and $0.1 \%$ SDS) and placed in the supporting tray, the tube was loaded at $100 \mathrm{~V}$ until the protein exited the gel slice using a mini-gel electrophoresis chamber (Mupid, ADVANCE CO., Ltd., Saitama, Japan). Next, reversing the polarity of the electric current for $120 \mathrm{sec}$, the protein-containing solution was transferred to a clean tube. Then, an equal volume of $20 \%$ TCA was added to the tube and it was incubated for $1 \mathrm{hr}$ at $4^{\circ} \mathrm{C}$, centrifuged at $18,000 \mathrm{x} \mathrm{g}$ for $30 \mathrm{~min}$ at $4^{\circ} \mathrm{C}$, and the supernatant was discarded. After adding cold acetone, the sample was incubated at $-20^{\circ} \mathrm{C}$ for $1 \mathrm{hr}$, and then centrifuged at $18,000 \mathrm{x} \mathrm{g}$ for $30 \mathrm{~min}$ at $4^{\circ} \mathrm{C}$. Finally, the supernatant was discarded, the pellet was air-dried, and Gal-3 protein was solubilized with detergent lysis buffer using an ultra-sonic homogenizer. ii)Recombinant Gal-3 protein: Recombinant Gal-3 protein was prepared using a wheat germ cell-free system (Goshima et al., 2008). Protein information and the sequence ID of recombinant Gal-3 protein are FLJ95838AAAF and G-BRTHA2025858_F.1, respectively (http://hgpd.lifesciencedb.jp/cgi/index.cgi).

\section{Dot blot analysis}

IgG levels of anti-Gal-3 autoantibody in sera were 
detected employing the automatic dot blot system, with a 256-solid pin configuration (Kakengeneqs Co., Ltd., Chiba, Japan). Gal-3 protein extracted by the electroelution method and recombinant protein were spotted onto the PVDF membranes. After being washed in TBS, the membranes were blocked with $2 \%$ Tween 20 in TBS for $1 \mathrm{hr}$ at RT. Then, the membranes were reacted with 1,000-times-diluted pre-treated sera with TBS-T for 16$18 \mathrm{hrs}$ at $4^{\circ} \mathrm{C}$. After washing 3 times for 5 min each in TBS-T, the membranes were incubated with 1,000-timesdiluted HRP-conjugated rabbit anti-human IgG polyclonal antibody (Dako) for $30 \mathrm{~min}$ at RT. Finally, signals were developed with Immobilon Western reagent (Millipore Corp.). The data were analyzed using DotBlotChipSystem software ver. 4.0 (Dynacom Co., Ltd., Chiba, Japan). Normalized signals are presented as the positive intensity minus background intensity around the spot. Statistical analysis was performed using the Mann-Whitney U-test. The area under the curve (AUC) and best cut-off point were calculated by employing receiver operating characteristics (ROC) analysis.

\section{Results}

Autoantigens identified by $2 D E-I B$

The proteins extracted from LC2/ad-cis cells were separated by 2-DE, transferred onto a PVDF membrane, and reacted with pre-treated sera from three patients who were assessed as showing PD or PR after platinumbased chemotherapy (Figure 1). Ninety-five and 63 immunoreactive spots were detected with pre-treated sera from AC patients who showed PD and PR, respectively. In total, 51 proteins including Gal-3 in $\mathrm{PD}$ and 12 proteins in PR patients, respectively, were identified (Table 2).

Gal-3 expressions in lung cancer cell lines

To confirm whether Gal-3 was over-expressed in
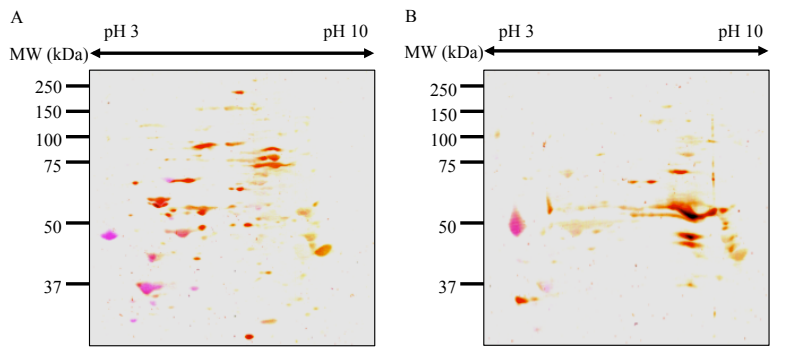

Figure 1. Detection of Autoantibodies in Pre-treated Sera from AC Patients who were Treated with Platinum-based Chemotherapy by 2DE-IB Analysis. Proteins extracted from LC2/ad-cis cells were separated by 2DE. Immunoblot analysis was performed with mixed pre-treated sera from AC patients assessed as showing PD (A) or PR (B) as a first-antibody, and the reaction was visualized with $\mathrm{DAB}$ solution

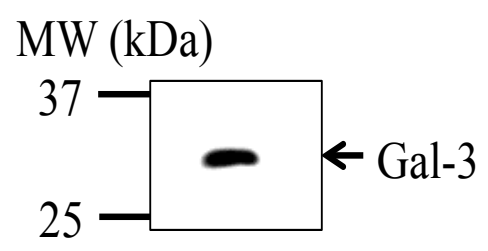

Figure 1. Results of Immunoblot Analysis
LC2/ad-cis cells compared to that in LC2/ad cells, we performed IB and IHC analysis using freshly frozen and formalin-fixed and paraffin-embedded LC2/ad cells and LC2/ad-cis cells, respectively (Figure 2). We confirmed that the expression of Gal-3 up-regulated in LC/2adcis cells and that positive staining was observed in the cytoplasm.

\section{Extraction of Gal-3 protein using electro-elution method}

We extracted Gal-3 protein from 2DE gels by the electro-elution method. The reactivity of extracted Gal-3 protein was confirmed by immunoblot analysis (Figure 1).

Different reactivity of anti-Gal-3 autoantibody to recombinant or extracted Gal-3 proteins

Anti-Gal-3 autoantibody in pre-treated sera from lung AC patients did not react with recombinant Gal-3 protein, but showed strong reactivity with Gal-3 protein extracted from tumor cells (Figure 3 ). Thus, the serum IgG levels of anti-Gal-3 autoantibody were validated by the dot blot analysis with extracted Gal-3 protein from tumor cells.

\section{Validation of anti-Gal-3 autoantibody}

To confirm its utility as a potential predictive biomarker of the efficacy of platinum-based chemotherapy against lung AC, we investigated IgG levels of anti-Gal-3 autoantibody in pre-treated sera by dot-blot analysis. The serum IgG levels of anti-Gal-3 autoantibody in PR, PD, and SD groups ranged from 190 to 1,070 (median: 550 ), 650 to 2,300 (median: 1,140 ), and 310 to 1,150 (median: 880), respectively. The serum IgG levels of anti-Gal-3 autoantibody were significantly higher in the PD group than in PR and SD groups $(\mathrm{p}=0.0084)$ (Figure 4 A). An optimal cut-off value of 1,086 was applied based on ROC analysis, and the predictive sensitivity and
A

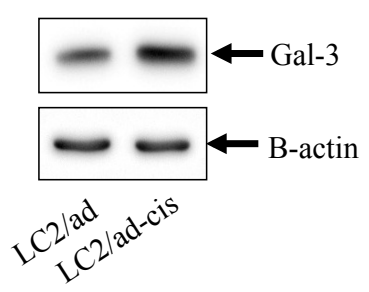

C

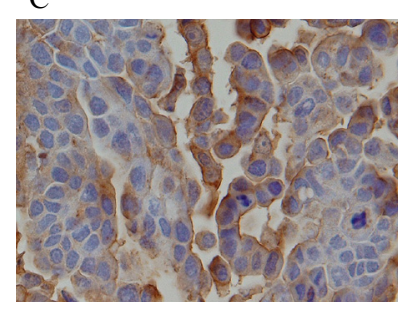

B

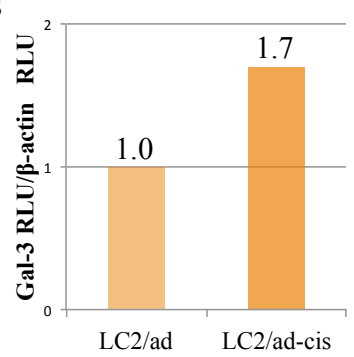

D

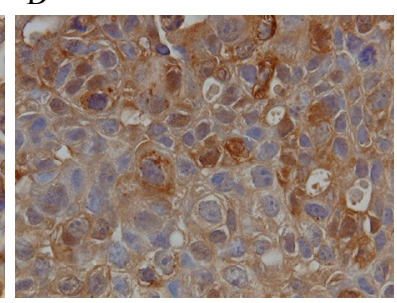

Figure 3. Expression of Gal-3 in Cisplatin-Resistant LC2/ad-cis Cells and Sensitive LC2/ad Cells. (A) Expression levels of Gal-3 protein were detected by immunoblot analysis. Beta-actin was used as an internal control. (B) Expression levels of Gal-3 were normalized by expression levels of beta-actin and presented as Relative Light Units (RLU). Cytoplasmic staining of Gal-3 in LC2/ad cells (C) and LC2/ ad-cis cells (D) 
Serum anti-Gal-3 Antibodies for Prediction of Efficacy of Platinum-based Chemotherapy against Pulmonary Adenocarcinoma

Table 2. Autoantigens Identified in Patients Assessed as Showing Partial Response or Progressive Disease

\begin{tabular}{|c|c|c|c|c|}
\hline \multicolumn{2}{|c|}{ Gene symbol Protein name } & IPI & \multicolumn{2}{|c|}{$\begin{array}{l}\text { Molecular } \\
\text { weight (Da) }\end{array}$} \\
\hline \multicolumn{5}{|c|}{ Partial Response } \\
\hline TUBA1A & Tubulin, alpha 1a & 00180675 & 50,136 & Major constituent of microtubules \\
\hline KRT8 & Keratin, type II cytoskeletal 8 & 00554648 & 53,704 & Links contractile apparatus to dystrophin at costameres of striated muscle \\
\hline AHCY & Adenosylhomocysteinase & 00012007 & 47,716 & $\begin{array}{l}\text { Competitive inhibitor of S-adenosyl-L-methionine-dependent methyl } \\
\text { transferase reactions" }\end{array}$ \\
\hline LRMP & Lymphoid-restricted membrane protein 1 & 00006158 & 62,122 & Developmentally regulated protein in lymphoid cell lines and tissues. \\
\hline MYH4 & Myosin-4 & 00001753 & 223,013 & Muscle contraction \\
\hline LMNA & Prelamin-A/C & & 74,139 & Accelerates smooth muscle cell senescence \\
\hline EEF1D & Eukaryotic translation elongation factor 1 delta & 00023048 & 31,122 & $\begin{array}{l}\text { EF-1-beta and EF-1-delta stimulate the exchange of GDP bound to EF- } \\
\text { 1-alpha to GTP }\end{array}$ \\
\hline HNRNPA1 & Heterogeous nuclear ribonucleoprotein A1 & 00215965 & 34,196 & Transport of poly(A) mRNA from the nucleus to the cytoplasm \\
\hline ACADM & Aacyl-CoA dehydrogenase, mitochondrial & 00005040 & 44,892 & enzyme specific for acyl chain lengths of 4 to 16 \\
\hline SFPQ & Isoform Long Splicing factor, proline-glutamine & 00010740 & 76,149 & DNA- and RNA binding protein, nuclear processes. \\
\hline DLST & Dihydrolipoyllysine-residue succinyltansferase & 00420108 & 48,755 & Multiple copies of 3 enzymatic components \\
\hline TUBA1B & tubulin, alpha $1 \mathrm{~b}$ & 00792677 & 50,152 & Tubulin is the major constituent of microtubules \\
\hline \multicolumn{5}{|c|}{ Progressive Disease } \\
\hline RDX & Radixin & 00017367 & 68,564 & $\begin{array}{l}\text { Crucial role in the binding of the barbed end of actin filaments to the } \\
\text { plasma membrane }\end{array}$ \\
\hline EZR & Ezrin & 00843975 & 69,413 & Connections of major cytoskeletal structures to the plasma membrane \\
\hline HSP90AA1 & Heat shock protein HSP 90 -alpha & 00784295 & 84,660 & Molecular chaperone that promotes maturation \\
\hline KARS & Lysyl-tRNA synthetase & 00014238 & 68,048 & Catalyzes specific attachment of amino acids to cognate tRNA \\
\hline PDIA4 & Protein disulfide isomerase family A, member 4 & 00009904 & 72,932 & Catalyzes the rearrangement of $-\mathrm{S}-\mathrm{S}$ - bonds in proteins \\
\hline TRAP1 & TNF receptor-associated protein 1 & 00030275 & 80,110 & Chaperone that expresses ATPase activity \\
\hline KRT19 & Keratin, type I cytoskeretal 19 & 00794644 & 44,106 & I nvolved in the organization of myofibers. \\
\hline HNRNPL & Heterogeous nuclear ribonucleoprotein $\mathrm{L}$ & 00027834 & 64,133 & A component of the hnRNP complexes \\
\hline KRT7 & Keratin, type II cytoskeretal 7 & 00792841 & 51,386 & Blocks interferon-dependent interphase and stimulates DNA synthesis \\
\hline KRT18 & Keratin, type I cytoskeretal 18 & 00554788 & 48,058 & Uptake of thrombin-antithrombin complexes by hepatic cells \\
\hline RUVBL2 & RuvB-like 2 & 00009104 & 51,157 & $\begin{array}{l}\text { Possesses single-stranded DNA-stimulated ATPase and ATP-dependent } \\
\text { DNA helicase (5' to 3') activity }\end{array}$ \\
\hline TUBB2C & Tubulin, beta $2 \mathrm{c}$ & 00956734 & 49,831 & Tubulin is the major constituent of microtubules \\
\hline TUBA1C & Tubulin, alpha 1c & 00218343 & 49,895 & Tubulin is the major constituent of microtubules \\
\hline SEPT11 & Septin-11 & 00019376 & 49,398 & Filament-forming cytoskeletal GTPase \\
\hline G6PD & Glucose-6-phosphate dehydrogenase & 00884082 & 59,257 & Produces pentose sugars \\
\hline EEF1A1 & Elongation factor 1-alpha 1 & 00180730 & 50,141 & $\begin{array}{l}\text { Promotes the GTP-dependent binding of aminoacyl-tRNA to the A-site } \\
\text { of ribosomes during protein biosynthesis }\end{array}$ \\
\hline IDH1 & Isocitrate dehydro genase cytoplasmic & 00027223 & 46,659 & Catalyzes the oxidative decarboxylation of isocitrate to 2-oxoglutarate. \\
\hline PGAM1 & Phosphoglycerate mutase 1 & 00549725 & 28,804 & $\begin{array}{l}\text { Interconversion of 3-and 2-phosphoglycerate with 2,3-bisphosphoglycerate } \\
\text { as the primer of the reaction }\end{array}$ \\
\hline SLC25A5 & ADP/ATP translocase 2 & 00007188 & 32,852 & Catalyzes exchange of ADP/ATP across the mitochondrial inner membrane \\
\hline PSMA1 & Proteaseme subunit alpha type-1 & 00910408 & 29,556 & ATP-dependent proteolytic activity \\
\hline EFHD2 & EF-hand domain-containing protein D2 & 00060181 & 26,697 & Regulate B-cell receptor induced immature and primary B-cell apoptosis \\
\hline PHB & Prohibitin & 00816719 & 29,804 & Prohibitin inhibits DNA synthesis \\
\hline LDHB & L-lactate dehydrogenase B & 00219217 & 36,638 & $\begin{array}{l}\text { Enzyme which catalyzes reversible conversion of lactate and pyruvate, } \\
\text { and NAD and NADH, in the glycolytic pathway. }\end{array}$ \\
\hline CAPZB & Isoform 2 of F-actin capping protein beta sub & 00642256 & 31,350 & F-actin-capping proteins bind \\
\hline GALE & UDP-galactose-4-epimerase & 00107100 & 38,282 & Catalyzes two distinct but analogous reactions \\
\hline BUB3 & Isoform 1 of mitotic checkpoint protein BUB 3 & 00013468 & 37,115 & $\begin{array}{l}\text { Dual function in spindle-assembly checkpoint signaling and promoting } \\
\text { the establishment of correct kinetochore-microtubule attachments. }\end{array}$ \\
\hline EIF2S1 & Eukaryotic translation initiation factor 2 , sub 1 & 00219678 & 36,112 & $\begin{array}{l}\text { Early steps of protein synthesis by forming a ternary complex with GTP } \\
\text { and initiator tRNA }\end{array}$ \\
\hline NQO1 & $\mathrm{NAD}(\mathrm{P}) \mathrm{H}$ dehydrogenase 1 & 00619966 & 30,868 & $\begin{array}{l}\text { Vit K-dependent gamma-carboxylation of glutamate residues in prothrombin } \\
\text { synthesis }\end{array}$ \\
\hline LGALS3 & Galectin-3 & 00385850 & 26,152 & $\begin{array}{l}\text { Acute inflammatory responses - neutrophil activation and adhesion, } \\
\text { chemoattraction of monocytes }\end{array}$ \\
\hline GNB2L1 & Guanine nucleotide binding protein sub beta 2 & 00848226 & 35,077 & Recruitment, assembly and/or regulation of signaling molecules \\
\hline HNRNPA2B1 & 1 Heterogeous nuclear ribonucleoprotein A2/B1 & 00414696 & 37,430 & pre-mRNA processing \\
\hline GAPDH & Glyceraldehyde-3-phosphate dehydrogenase & 00219018 & 36,053 & Glyceraldehyde-3-phosphate dehydrogenase and nitrosylase activities \\
\hline ALDOA & Fructose-bisphosphate aldolase A & 00465439 & 39,420 & Glycolysis and gluconeogenesis \\
\hline PSMC6 & $26 \mathrm{~S}$ protease regulatory subunit $\mathrm{S} 10 \mathrm{~B}$ & 00910829 & 44,173 & ATP-dependent degradation of ubiquitinated proteins \\
\hline $\mathrm{FH}$ & Fumarate hydratase & 00759715 & 54,637 & Acts as a tumor suppressor \\
\hline ENO1 & Enolase 1 & 00465248 & 47,169 & Multifunctional enzyme \\
\hline ANXA11 & Annexin A11 & 00414320 & 54,390 & Produces pentose sugars \\
\hline UGDH & UDP-glucose 6-dehydrogenase & 00968010 & 55,024 & Biosynthesis of glycosaminoglycans \\
\hline GSR & Isoform Mitochondrial of Glutathione reductase & 00016862 & 56,257 & Binds specifically to calcyclin \\
\hline SHMT2 & Serine hydroxymethyltransferase 2 & 00794572 & 55,993 & Interconversion of serine and glycine \\
\hline СCT7 & T-complex protein 1 subunit 7 & 00952607 & 59,367 & Folding of proteins upon ATP hydrolysis \\
\hline DDX6 & Probable ATP-dependent RNA helicase DDX6 & 00030320 & 54,417 & mRNA decapping \\
\hline DDX5 & Probable ATP-dependent RNA helicase DDX5 & 00017617 & 69,148 & RNA-dependent ATPase activity \\
\hline OGG1 & 8-oxoguanine DNA glycosylase & 01013811 & 38,782 & DNA repair enzyme that incises DNA at 8-oxoG residues. \\
\hline AIFM1 & Apoptosis-inducing factor 1 & 01015707 & 66,901 & Controlling cellular life and death \\
\hline KRT10 & Keratin, type I cytoskeletal 10 & 00009865 & 58,827 & $\begin{array}{l}\text { This gene encodes a member of the type I (acidic) cytokeratin family, } \\
\text { which belongs to the superfamily of intermediate filament (IF) proteins. }\end{array}$ \\
\hline tmpo & Thymopoietin & 00216230 & 75,492 & Structural organization of the nucleus \\
\hline NCL & Nucleolin & 00916991 & 76,614 & Nucleolar protein of growing eukaryotic cells \\
\hline KHSRP & Far upstream element-binding protein & 00855957 & 73,115 & Dendritic targeting element \\
\hline UBA1 & Ubiquitin-like modifier activating enzyme 1 & 00645078 & 117,849 & Activates ubiquitin \\
\hline DDX1 & Probable ATP-dependent RNA helicase DDX1 & 00293655 & 82,432 & ATP-dependent RNA helicase \\
\hline
\end{tabular}




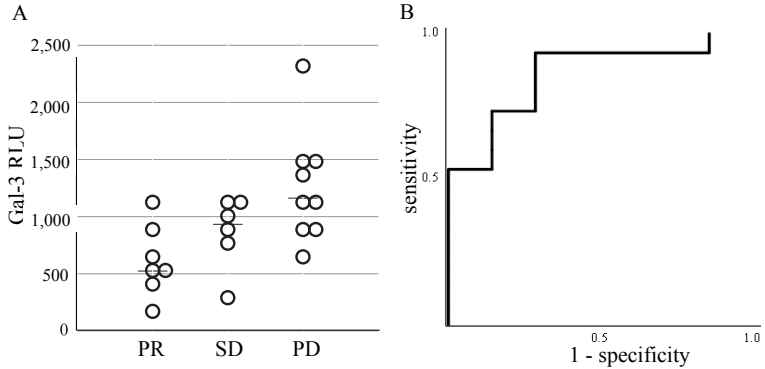

Figure 4. Anti-Gal-3 Autoantibody Levels in PreTreated Sera from Lung Cancer Patients by Dot-blot Analysis. (A) The median Gal-3 autoantibody level in pretreated sera from patients assessed as showing PR and SD or PD was 873 or 1,140 . Anti-Gal-3 autoantibody levels were significantly higher in PD patients than PR and SD patients ( $p$ $<0.0084$ ). (B) Receiver operating characteristic curve (ROC) analysis of serum anti-Gal-3 autoantibody levels as a predictive marker of the efficacy of platinum-based chemotherapy against lung adenocarcinoma. The corresponding area under the curve was 0.84 for anti-Gal-3 autoantibody. With $92 \%$ specificity, the sensitivity of anti-Gal-3 autoantibody for patients assessed as showing PD was $67 \%$, at a cut-off value corresponding to 1,086

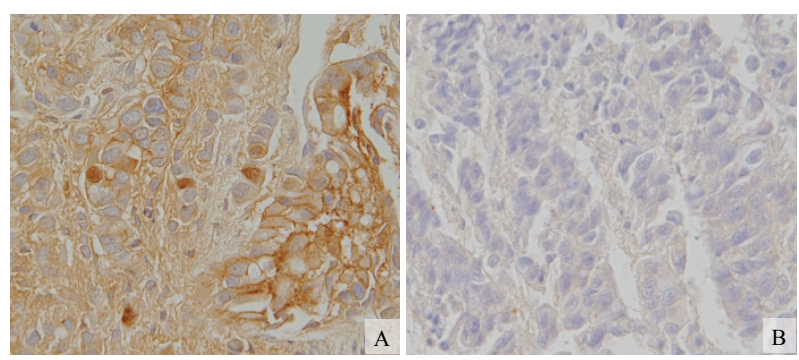

Figure 5. Expression of Gal-3 in lung cancer biopsy samples. (A) The section from patients who assessed as showing progressive disease (PD) following platinum-based chemotherapy was evaluated as Gal-3-positive. (B) The section from patients who assessed as showing partial response (PR) to platinum-based chemotherapy was evaluated as Gal-3-negative

specificity for platinum-based chemotherapy was 67 and $92 \%$, respectively (Figure $3 \mathrm{~B}$ ). The AUC for anti-Gal-3 autoantibody in patients who showed PD compared to $\mathrm{SD}$ and PR after platinum-based chemotherapy was 0.84 .

\section{Gal-3 expression in lung cancer biopsy samples}

To further clarify the relation between Gal-3 expression and the efficacy of platinum-based chemotherapy, we compared the stainability and clinical data in 40 pretreated lung biopsy samples. We judged the Gal-3 staining as negative when the staining score was 0 or 1 (Figure $5 \mathrm{~A}$ ), while it was positive when the score was 2 or 3 (Figure 5 B). Gal-3 expression was evaluated as positive in 10 of 15 (66.7\%) PD, 7 of $14(50.0 \%) \mathrm{SD}$, and in 2 of $11(18.2 \%)$ PR patients, respectively. The positive rate of Gal-3 was in the order of PD, SD, and PR groups $(\mathrm{p}=0.052)$.

\section{Discussion}

In the present study, to identify novel biomarkers that can predict the efficacy of platinum-based chemotherapy, we performed $2 \mathrm{DE}-\mathrm{IB}$ to detect autoantigens that are recognized by autoantibodies in pre-treated sera from lung AC patients assessed as showing PD or PR often platinum-based chemotherapy. Sixty-three autoantigens in total were identified by 2DE-IB.

We focused on Gal-3 from the identified candidate autoantigens, because it was reported that intracellular Gal-3 exhibits activity to suppress drug-induced apoptosis, which was reported to be essential for cancer cell survival and tumor progression in various tumor types (Tsuboi et al., 2007, Park et al., 2008, Saussez et al., 2008, Chiu et al., 2010) and Gal-3 thus has been proposed as an antiapoptotic factor in response to chemotherapeutic drugs, and its down-regulation could improve the efficacy of anticancer drugs (Fukumori et al., 2006). However, to our knowledge, there has been no study on the utility of Gal-3 as an anti-apoptotic factor in lung cancer. They also reported that the expression of Gal-3 protein stimulated the phosphorylation of Ser112 of Bcl-2associated death (Bad) protein and down-regulated Bad expression after treatment with cisplatin. And, Gal-3 also inhibited mitochondrial depolarization and damage after translocation from the nuclei to cytoplasm, resulting in the inhibition of cytochrome $c$ release and caspase- 3 activation (Fukumori et al., 2006). These study suggest that Gal-3 inhibits drug-induced apoptosis through the phosphorylation of Bad protein and suppression of the mitochondrial apoptosis pathway.

Cisplatin-induced apoptosis was increased after knocking down the expression of Gal-3, and this cytotoxic effect was canceled by roscovitine, an inhibitor of cyclindependent protein kinase (Oishi et al., 2007). Wongkham et al. (Wongkham et al., 2009) reported that the cellular levels of Gal-3 might contribute to the anti-apoptotic activity and chemoresistance of cholangiocarcinoma cells. In the present study, the expression of Gal-3 was up-regulated in cisplatin-resistant LC2/ad-cis cells compared with the parental LC2/ad cells, consistent with previous reports (Fukumori et al., 2006, Oishi et al., 2007). Although the expression levels of Gal-3 in tumor cells have been reported to be correlated with in cisplatin resistance, the association between serum IgG levels of anti-Gal-3 autoantibody and cisplatin resistance has not been investigated.

To confirm the possibility of using anti-Gal-3 autoantibody as a novel predictive marker of the efficacy of platinum-based chemotherapy, we measured the serum anti-Gal-3 autoantibody levels in patients with diseases assessed as showing PR, SD, or PD following platinumbased treatment by dot-blot analysis. We demonstrated that the serum anti-Gal-3 autoantibody levels were significantly higher in the PD group than in PR and $\mathrm{SD}$ groups $(\mathrm{p}=0.0084)$. Furthermore, IHC staining of pretreated lung biopsy samples also showed that the positive rate of Gal-3 was highest in the PD group, and was decreased in the order of PD, SD, and PR groups $(\mathrm{p}=0.052)$. Our results confirmed that the expression of Gal-3 had a role in the anti-apoptotic activity and chemoresistance. Taken together, our results suggest the serum anti-Gal-3 autoantibody levels might be a useful predictive marker of the efficacy of platinum-based chemotherapy.

The different reactivity between recombinant and extracted Gal-3 proteins might be caused by the presence 
Serum anti-Gal-3 Antibodies for Prediction of Efficacy of Platinum-based Chemotherapy against Pulmonary Adenocarcinoma or absence of post-translational modifications (PTM) (Balan et al., 2010). The present data raise the possibility that anti-Gal-3 autoantibody in sera from lung AC patients reacts with the modification-received Gal-3 protein, and further studies are necessary to investigate this hypothesis.

In conclusion, we demonstrated that IgG levels of antiGal-3 autoantibody in pre-treated sera were significantly higher in patients assessed as showing PD than as PR and SD after platinum-based chemotherapy. Our data suggest that the IgG levels of anti-Gal-3 autoantibody provide a novel predictive biomarker of the efficacy of platinumbased chemotherapy.

\section{Acknowledgements}

This study was supported in part by JSPS KAKENHI Grant Number 23590414 (to Y. S.), the JST-SENTAN (Development of Systems and Technology for Advanced Measurement and Analysis: Life Innovation Area) program from the Japan Science and Technology Agency (to N. G. and Y. S.), the 2013-2014 Project Study from the Graduate School of Medical Sciences, the Research Project (No. 2013-1004) from the School of Allied Health Sciences, Kitasato University to Y. S.), and a grant-in-aid of The Ishidsu Shun Memorial Scholarship, Japan (to K. Y.).

\section{References}

Balan V, Nangia-Makker P, Jung YS, et al (2010). Galectin-3: a novel substrate for c-Abl kinase. Biochem Biophys Acta, 1803, 1198-205.

Chang A (2011). Chemotherapy, chemoresistance and the changing treatment landscape for NSCLC. Lung Cancer, 7,3-10.

Chiu CG, Strugnell SS, Griffith OL, et al. Diagnostic utility of galectin-3 in thyroid cancer. Am J Pathol, 176, 2067-81.

Fernández-Madrid F, Tang N, Alansari H, et al (2004). Autoantibodies to Annexin XI-A and other autoantigens in the diagnosis of breast cancer. Cancer Res, 64, 5089-96.

Fukumori T, Oka N, Takenaka Y, et al. Galectin-3 regulates mitochondrial stability and antiapoptotic function in response to anticancer drug in prostate cancer. Cancer Res, 66, 3114-9.

Gonzalez V M, Fuertes M A, Alonso C, et al (2001). Is cisplatininduced cell death always produced by apoptosis? Mol Pharmacol, 59, 657-63.

Goshima N, Kawamura Y, Fukumoto A, et al. Human protein factory for converting the transcriptome into an in vitroexpressed proteome. Nat Methods, 5, 1011-7.

Hanash S (2003). Harnessing immunity for cancer marker discovery. Nat Biotechnol, 21, 37-8.

Jemal A, Siegel R, Ward E, et al (2007). Cancer statistics, 2007. CA Cancer J Clin, 57, 43-66.

Kageyama T, Nagashio R, Ryuge S, et al (2011). HADHA is a potential predictor of response to platinum-based chemotherapy for lung cancer. Asian Pac J Cancer Prev, 12, 3457-63.

Laemmli UK (1970). Cleavage of structural proteins during the assembly of the head of bacteriophage T4. Nature, 227, 680-5.

Le Naour F, Brichory F, Misek DE, et al (2002). A distinct repertoire of autoantibodies in hepatocellular carcinoma identified by proteomic analysis. Mol Cell Proteomics, $\mathbf{1}$, 